\title{
Review of: "COVID-19 vaccination and unemployment risk: lessons from the Italian crisis"
}

corrado ciatti

Potential competing interests: The author(s) declared that no potential competing interests exist.

Introduction section is quite long. Try to reduce it, also giving a precise order to it, providing first a background and in the end of tue section the aim of the study. Add more references to this part. Materials and methods section have to follow the introduction section.

When writing "results" try to not provide any comment, but only report data.

Discussion is too short and poor. In addiction, this section should conteins more references which support your findings.

In the "conclusions" section you should be more concise, reporting only the findings of this study. All the other information have to be moved to the discussion.

References ok.

Figures ok

Language: minor revision. 